\section{A) Check for updates}

Cite this: Nanoscale, 2021, 13, 16324

\title{
Multifunctional superparamagnetic nanoparticles with a fluorescent silica shell for the in vitro study of bio-nano interactions at the subcellular scale $\uparrow$
}

\author{
Lorenzo Cursi, (D) $\ddagger^{a}$ Silvia Vercellino, (D) $\ddagger^{a, b}$ Mura M. McCafferty, (D) a \\ Emily Sheridan, (D) ${ }^{a}$ Vanya Petseva, (D) a Laurent Adumeau (D) *a and \\ Kenneth A. Dawson (D) *a
}

\begin{abstract}
Despite the high level of interest in bio-nano interactions, detailed intracellular mechanisms that govern nanoscale recognition and signalling still need to be unravelled. Magnetic nanoparticles (NPs) are valuable tools for elucidating complex intracellular bio-nano interactions. Using magnetic NPs, it is possible to isolate cell compartments that the particles interact with during intracellular trafficking. Studies at the subcellular scale rely heavily on optical microscopy; therefore, combining the advantages of magnetic recovery with excellent imaging properties to allow intracellular NP tracking is of utmost interest for the nanoscience field. However, it is a challenge to prepare highly magnetic NPs with a suitable fluorescence for the fluorescence imaging techniques typically used for biological studies. Here we present the synthesis of biocompatible multifunctional superparamagnetic multicore NPs with a bright fluorescent silica shell. The incorporation of an organic fluorophore in the silica surrounding the magnetic multicore was optimised to enable the particles to be tracked with the most common imaging techniques. To prevent dye loss resulting from silica dissolution in biological environments, which would reduce the time that the particles could be tracked, we added a thin dense encapsulating silica layer to the NPs which is highly stable in biological media. The synthesised multifunctional nanoparticles were evaluated in cell uptake experiments in which their intracellular location could be clearly identified using fluorescence imaging microscopy, even after 3 days. The magnetic properties of the iron oxide core enabled both efficient recovery of the NPs from the intracellular environment and the extraction of cell compartments involved in their intracellular trafficking. Thus, the NPs reported here provide a promising tool for the study of the processes regulating bio-nano interactions.
\end{abstract}

Received 14th July 2021,

Accepted 30th August 2021

DOI: $10.1039 / \mathrm{d} 1 \mathrm{nr} 04582 \mathrm{~b}$

rsc.li/nanoscale enzyme immobilization, ${ }^{4,5}$ targeted drug delivery, ${ }^{6,7}$ MRI enhancement, ${ }^{6,7}$ hyperthermia treatment, ${ }^{8-11}$ and point of care devices, ${ }^{12}$ owing to their unique magnetic properties. In biology, magnetic separation is often used in purification, ${ }^{13}$ in which the object of interest is labelled with a magnetic particle and manipulated by the application of a magnetic field. ${ }^{2}$ Recently, a number of studies have exploited magnetic particles for the isolation of cells, ${ }^{14}$ viruses, ${ }^{15,16}$ organelles, ${ }^{13}$ exosomes ${ }^{17,18}$ and other cell compartments. ${ }^{2,13}$ This ability to selectively recover superparamagnetic NPs from biological environments means they are becoming an essential tool for unravelling the interactions between nanoparticles and cells. ${ }^{2,13}$

Because they allow the same nanoparticle to be used throughout the experimental workflow enabling the direct correlation of the information collected during each stage, multifunctional nanoparticles ${ }^{19-21}$ are an important development in resolving the complexity of these bio-nano interactions. Fluorescent NPs are widely used to study bio-nano interactions as they offer multiple advantages for tracking NP behaviour in

\footnotetext{
Belfield, Dublin 4, Ireland. E-mail: laurent.adumeau@cbni.ucd.ie,

Kenneth.a.dawson@cbni.ucd.ie

${ }^{b}$ UCD Conway Institute of Biomolecular and Biomedical Research, School of Biomolecular and Biomedical Science, University College Dublin, Belfield, Dublin 4, Ireland

$\dagger$ Electronic supplementary information (ESI) available. See DOI: 10.1039/ d1nr04582b

\$Lorenzo Cursi and Silvia Vercellino contributed equally to this manuscript.
} 
the intracellular environment and offer an alternative to other destructive techniques. For example, optical imaging is often used to observe the intracellular trafficking of nanomaterials, ${ }^{22,23}$ while fluorescence measurement in flow cytometry can be used to quantify cellular uptake and other aspects of cell response. ${ }^{24}$ As such, combining magnetic properties with fluorescence within a single nanoparticle would provide a valuable tool for the study of bio-nano interactions as it allows one to directly correlate what is observed by microscopy with the molecular analysis of the cellular compartments magnetically extracted with the NPs. We next consider how to design such a tool.

For applications involving magnetic extraction, structures with a core made of several superparamagnetic NPs coated by a polymer have proven to outperform single small superparamagnetic NPs. ${ }^{25}$ By forming such multicore structures, the superparamagnetic properties of the single NPs are retained by the whole nanoconstruct. ${ }^{26}$ Multicores can be extracted more efficiently as they are less susceptible to Brownian motion and their magnetic force corresponds to the sum of the forces experienced by the single superparamagnetic particles. Iron oxide superparamagnetic NPs (SPIONs) are particularly interesting due to their simple synthesis, ease of functionalization and high magnetic susceptibility. ${ }^{27}$

There are several challenges to consider when designing NPs to obtain robust and reliable results based on fluorescence in biological studies. The NP signal must be clearly distinguishable from cellular autofluorescence to avoid phototoxicity from exposure to high laser intensity, common in the case of low NP fluorescence emission. Dye leakage should be eliminated as far as possible as this can lead to a diffuse signal that decreases over time and affects cell viability; poor photostability should be avoided as this precludes live cell imaging due to photobleaching. Although these challenges are now considered trivial for some transparent materials such as silica or polystyrene, they are not as easily overcome when the nanoparticle incorporates strongly absorbing materials such as metals or metal oxides.

Covalent incorporation of fluorescent organic molecules into silica NPs is easily accomplished through the modification of silicate derivatives. ${ }^{28,29}$ Moreover, silica is widely used in the fabrication of NPs and in the growth of shells on NPs because its synthesis allows both fine control over size and versatile surface modifications. It is also considered to be biocompatible and can help to reduce the toxicity of some metallic NPs. $^{30}$

Magnetic NPs coated with silica have been previously reported; however, these studies have predominantly involved small, single magnetic core NPs, ${ }^{31}$ or NPs with few cores incorporated within a thick silica matrix, ${ }^{32,33}$ making it difficult to magnetically extract them with the required efficiency for biological applications. To the best of our knowledge, there are few reports of synthesis of magnetic multicores loaded with a large number of SPIONs that are also highly fluorescent. Chen et al. report the synthesis of magneto-fluorescent nanoparticles combining the prospect of magnetic manipulation/ extraction of the nanoparticles, owing to the magnetic multicore, with bright fluorescence provided by the co-assembled quantum dots. ${ }^{34}$ However, quantum dots are known to be toxic and their synthesis complex. We were interested in using conventional organic fluorescent dyes instead of quantum dots but obtaining bright nanoparticles while also limiting fluorescence loss over time poses challenges. It is particularly important to load sufficient dye to ensure the particles are bright enough for the desired applications. On the other hand, overloading the silica matrix with organic dyes can cause quenching, mainly due to Förster resonance energy transfer (FRET) effects ${ }^{35,36}$ caused by the typically small Stokes shift of the organic fluorophores, but also due to agglomeration-caused quenching (ACQ) and proximity-caused quenching (PCQ). ${ }^{37}$

Dye leakage, an often neglected issue, can occur due to the dissolution of amorphous silica coatings in aqueous solutions and represents a serious limitation, especially in biological applications where the combination of a slightly alkaline $\mathrm{pH}$ and a relatively high salt concentration of the biological media has been shown to accelerate this process. ${ }^{38}$ To prevent dye loss, NPs can be coated with a protective, denser silica layer. Such a layer is usually synthesised with the aid of amino acids, such as arginine, that catalyse the synthesis of a denser layer of silica. ${ }^{39}$ However, amino acids can also adsorb on the surface of the NPs, altering their surface properties and subsequently interfering with their intracellular trafficking.

Here we present the design of a multifunctional core-shell nanoconstruct composed of a cluster of SPIONs, encapsulated in a fluorescent amorphous silica layer and protected with a thin layer of denser silica to prevent leakage of the fluorophore in biological media. The loading of fluorescein, an inexpensive organic dye, has been optimised to deliver maximum brightness of the core-shell structure. We also propose a mechanism of formation and a mechanism of action for the protective layer. We demonstrate that these nanoparticles can be tracked inside cells using classical and high-resolution fluorescence microscopy, and that the nanoparticles can be magnetically isolated after their journey within the cells. As such, we provide a tool with great potential for the study of bio-nano interactions at the subcellular level.

\section{Results and discussion}

\section{Dye loading in the silica matrix}

The synthesis of the magnetic multicore for encapsulation in the thin silica shell was adapted from a previously published procedure. ${ }^{40}$ Multicores composed of magnetite $\left(\mathrm{Fe}_{3} \mathrm{O}_{4}\right)$ NPs were compared to multicores composed of maghemite $\left(\gamma-\mathrm{Fe}_{2} \mathrm{O}_{3}\right)$ NPs. Both multicores were estimated to be around 50-60 $\mathrm{nm}$ in diameter by TEM imaging (see ESI Fig. S1 and $\mathrm{S} 2 \uparrow$ for characterisation).

Silica is particularly well adapted for fluorescence labelling because it is transparent in the visible light domain. However, the presence of non-transparent cores, as in the case of iron oxide NPs presenting strong absorbance in the visible region 
of light, impacts the brightness of the particles. Therefore, when designing such core-shell structures, the optimization of dye loading to simultaneously maximize the NPs fluorescence and minimize quenching effects is particularly important.

Organic dyes can be incorporated into the silica matrix by two different processes: physical entrapment in the matrix or covalent conjugation to the matrix. ${ }^{30}$ The latter is preferred as it reduces dye leaking. It generally requires the dye to be conjugated to a functional organosilane (Fig. 1a) which is then covalently bound to the silica matrix. ${ }^{29,41}$ Many different commercially available functional organic dyes can be incorporated into the silica matrix depending on the desired application and the available excitation and emission channels of the instrument in question.

In this study, to illustrate the versatility of the process, we used two different conjugation chemistry methods to prepare the dyes for incorporation into the silica matrix and we also varied the fluorophores. The first route involved the use of (3-aminopropyl)trimethoxysilane (APTMS) to activate two different dyes: Rhodamine $\mathrm{B}$ isothiocyanate (RB) and fluorescein isothiocyanate (FITC). The other employed (3-mercaptopropyl)trimethoxysilane (MPTMS) to react with the maleimide function in each of Cyanine 3-, Cyanine 5- and Cyanine 7-maleimide (Cy3, Cy5 and Cy7 respectively) and fluorescein maleimide (FM) dyes. The resulting fluorophore-APTMS or fluorophore-MPTMS adducts were then reacted with tetraethyl orthosilicate (TEOS) in a basic hydro-alcoholic media (via the Stöber process) to covalently incorporate them into the silica matrix. The synthesis led to bright $\mathrm{SiO}_{2}$ NPs with a fluorescence emission ranging the whole spectrum of visible light (Fig. 1b). The organic dyes incorporated into the silica matrix with this strategy retain excitation and emission spectra profiles similar to their free forms (as reported by the supplier).

For the optimization of the fluorescence labelling of the silica shell, we decided to use FITC as it is one of the most inexpensive organic dyes frequently used for labelling NPs for biological studies. To optimise the labelling, plain $\mathrm{SiO}_{2} \mathrm{NPs}$ of about $60 \mathrm{~nm}$ (similar size and size distribution to iron oxide cores, ESI Fig. S3†) were used in place of the magnetic multicore NPs to eliminate any iron oxide core influence on the fluorescence measurements.

The first parameter we optimised was the excess of APTMS to use per FITC in order to maximise the incorporation of the dye in the silica matrix.

a.

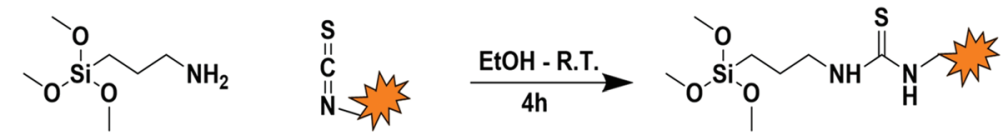

(3-Aminopropyl)trimethoxy Fluorophore silane Isothiocyanate

Activated Fluorophore
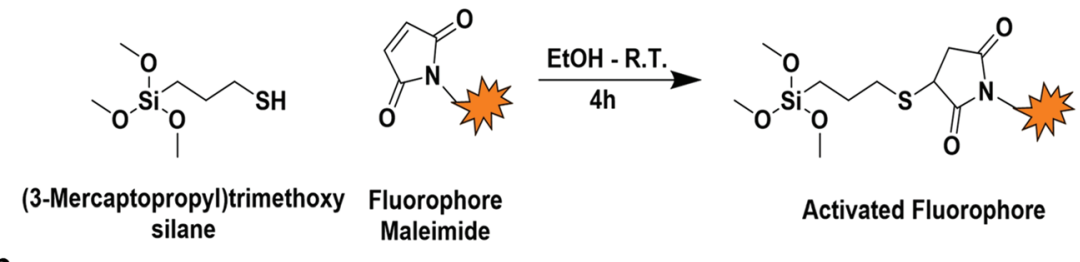

b.

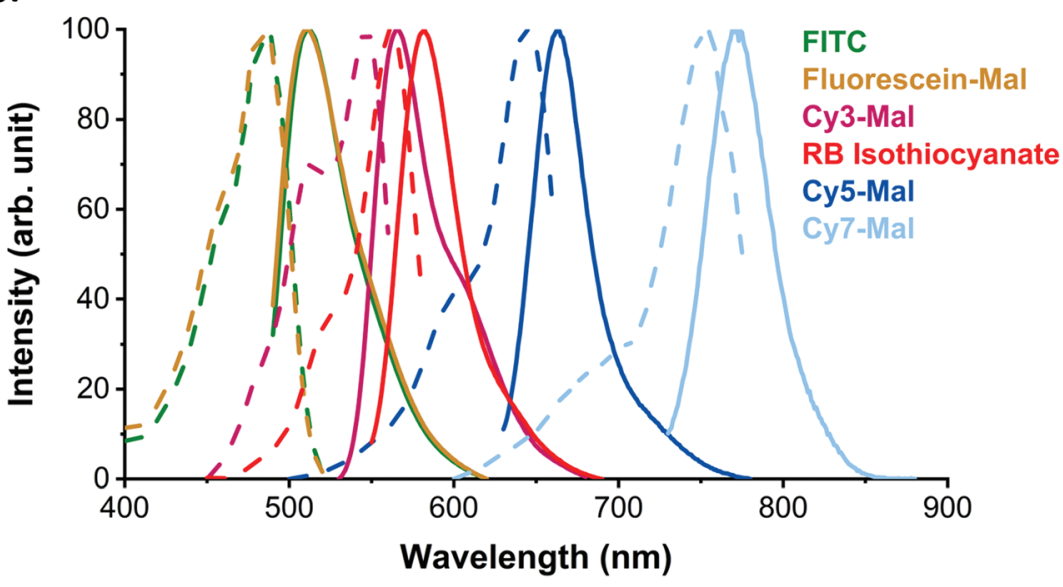

Fig. 1 Incorporation of organic dyes into $\mathrm{SiO}_{2} \mathrm{NPs}$. (a) Scheme of reaction between the organic dye (with thiocyanate or maleimide functions) and the respective amino- or mercaptosilane. The product of these reactions then reacts with TEOS under Stöber conditions. (b) The excitation (dotted line) and emission (solid line) spectra of $\mathrm{SiO}_{2} \mathrm{NPs}$ doped with different organic fluorophores. The spectra of the incorporated dyes are similar to the spectra of their free forms (as reported by suppliers). 
FITC was activated, in ethanol, with an excess of APTMS ranging from 1 to 30 equivalents. After the reaction with APTMS, the dye was incorporated into the silica shell grown over the $60 \mathrm{~nm}$ plain- $\mathrm{SiO}_{2}$ core. The dye incorporation was evaluated by measuring the absorbance of the NP dispersion in the visible region.

The amount of dye incorporated in the NPs (calculated as described in ESI methods, Fig. $\mathrm{S} 4 \dagger$ ) reached a plateau at an APTMS : FITC ratio of 10:1 (Fig. 2a). However, using a larger excess appears to improve the fluorescence of the NPs (ESI Fig. S5†) by increasing the fluorescence efficiency of the FITC (Fig. 2b). To explain this observation, we suggest that ACQ plays a major role in quenching the fluorescence when insufficient APTMS excess is present to activate the FITC. ${ }^{37}$ Indeed, it is known that APTMS can spontaneously form oligomers in the presence of water (even trace amounts) as the amine autocatalyzes the hydrolysis of the methoxy groups, as well as the condensation of the formed silanolates. When a molecule of FITC binds to an oligomer to which another molecule of FITC is already bonded too closely, they can undergo ACQ and FRET. Increasing the excess of APTMS, may increase the (average) length of the oligomers formed or promote the formation of a larger number of oligomers. In both cases, there is a smaller probability that two dye molecules find themselves too close to each other in the same chain and so quenching is reduced. Increasing the APTMS : FITC ratio is then beneficial for the fluorescence of the particles; however, the presence of aminosilanes in the silica matrix can affect the hydrolysis rate of the silica. In some cases, the aminosilane will reduce the dissolution rate of the silica thanks to a combination of factors including the inductive donor effect of the alkyl chain reducing the electropositive character of Si centre, and an increase in hydrophobicity. ${ }^{42,43}$ In other cases, the presence of aminosilane has been reported to increase the dissolution rate of the silica, possibly due to the presence of amine groups catalysing the hydrolysis as well as the decrease of the condensation of the silica network. ${ }^{29,39}$ For these reason and because the fluo- rescence increase remains small (increase of $15 \%$ at best), we decided to use only an excess of 10 equivalent of APTMS per dye. While not observed here, large excess of APTMS in different conditions have also been associated to the destabilization of the silica growth leading to aggregation ${ }^{39,44}$

Following the activation of the dye, its incorporation into the silica matrix is also crucial. It has been reported that overloading NPs with FITC leads to ACQ and PCQ as well as FRET. ${ }^{36}$ To evaluate the optimal amount of dye to incorporate into the silica matrix, we trialled different amounts of FITC (activated using 10 equivalents of APTMS) based on the \% weight versus TEOS used in silica growth. The loading of FITC in the $\mathrm{SiO}_{2} \mathrm{NPS}$ increased linearly (Fig. 3a), while their fluorescence peaked when FITC-APTMS was used at $0.5 \% \mathrm{w} / \mathrm{w}$ versus TEOS, and then decreased because of quenching effects (Fig. 3b).

The percentage of FITC was calculated based on the amount added during the synthesis and not from the amount of fluorophore actually incorporated. Indeed, we found that despite a complete functionalisation of the FITC with 10 equivalents of APTMS, according to HPLC (ESI Fig. S6†), the incorporation of the dye was only 50-60\% effective (ESI Fig. S6†). We attribute this result to the instability of the thiourea bond in alkaline solutions. Considering that the maximum fluorescence efficiency was obtained for the sample loaded with $0.5 \% \mathrm{w} / \mathrm{w}$, we estimated that an average distance in the range of 5.8 to $6.9 \mathrm{~nm}$ between molecules of FITC is necessary before the quenching dominates over the benefit of the loading increase (ESI methods, Fig. S7, 8 and Table S2 $\dagger$ ).

The optimised parameters for the fluorescence labelling were then applied to the maghemite and magnetite multicore $\left(\gamma-\mathrm{Fe}_{2} \mathrm{O}_{3} @ \mathrm{SiO}_{2}\right.$ FITC and $\mathrm{Fe}_{3} \mathrm{O}_{4} @ \mathrm{SiO}_{2}$ FITC respectively) and plain silica core $\left(\mathrm{SiO}_{2} @ \mathrm{SiO}_{2}\right.$ FITC) NPs.

The presence of different types of cores affects both the absorbance and fluorescence of the NPs (Fig. 3c, ESI Fig. S9-11†). The fluorescence intensity of the dispersion is inversely related to the absorbance of the cores in the range of the excitation and emission of the fluorophore. In measuring a.

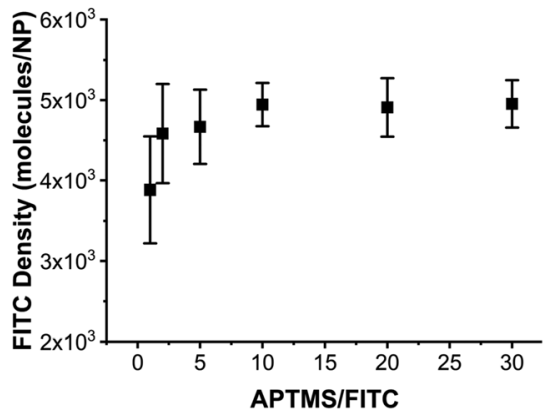

b.

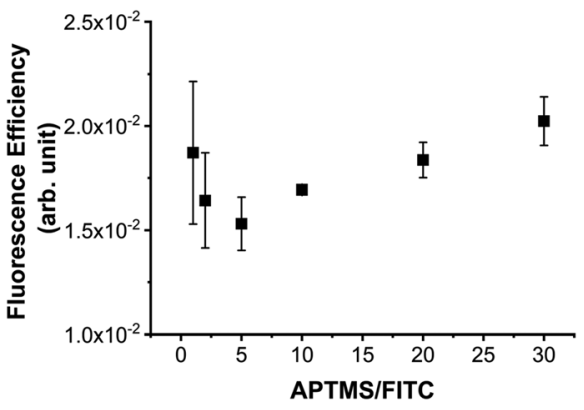

Fig. 2 Optimization of FITC activation with APTMS. (a) Average FITC density (molecules/NP), the error bars indicate the standard error on the three replicates, and (b) fluorescence efficiency of the fluorophore incorporated in the fluorescent silica layer grown on the $60 \mathrm{~nm}$ plain-SiO $\mathrm{O}_{2}$ core, as measured over three sets of independent experiments. The error bars indicate the standard error on the three replicates. Activating FITC with different excesses of APTMS. The incorporation of FITC reach a plateau when at least 10 equivalents of APTMS are used for the activation of FITC, but larger excess improves the fluorescence efficiency. 
a.

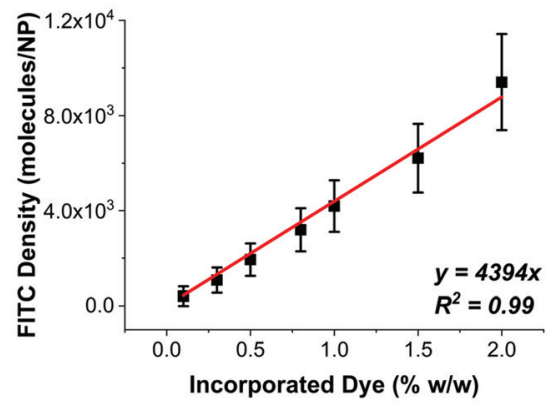

b.

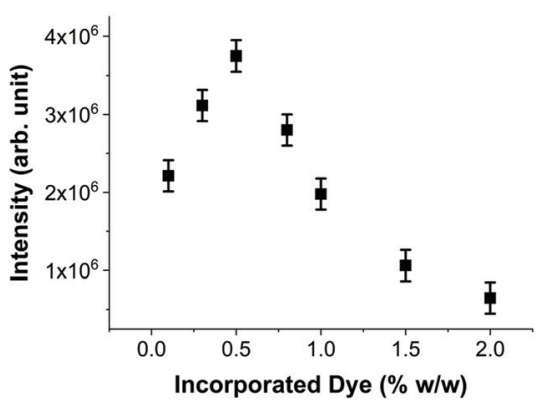

c.

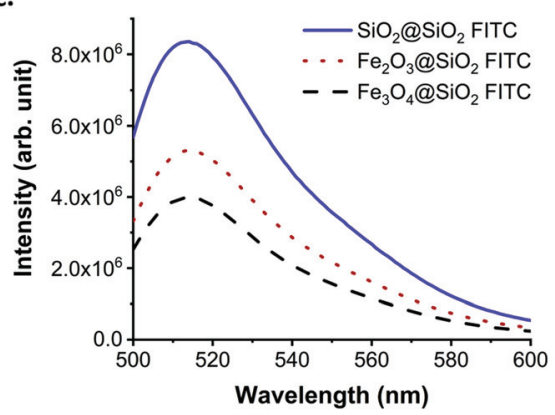

Fig. 3 Effect of dye loading and core material on the fluorescence. (a) FITC density (molecules/NP). The error bars correspond to the sum of the instrumental and experimental error. (b) Fluorescence of the silica layer grown on the $60 \mathrm{~nm}$ plain-SiO ${ }_{2}$ core, using different amounts of FITCAPTMS. The error bars correspond to the instrumental error. While the amount of incorporated dye increases linearly, the fluorescence reaches a maximum and then decreases due to $A C Q, P C Q$ and FRET effects. (c) Fluorescence spectra of $\mathrm{SiO}_{2} \mathrm{QSiO}_{2} \mathrm{FITC}_{1} \gamma-\mathrm{Fe}_{2} \mathrm{O}_{3}\left(\mathrm{QSiO} \mathrm{O}_{2} \mathrm{FITC}\right.$ and $\mathrm{Fe}_{3} \mathrm{O}_{4}\left(\mathrm{QSiO}_{2} \mathrm{FITC} \mathrm{NPs}\right.$ at the same concentration $\left(4 \times 10^{10} \mathrm{NPs}\right.$ per $\left.\mathrm{mL}\right)$. The presence of the iron oxide cores affects the fluorescence of the coreshell structure absorbing the light emitted from the fluorophore.

the fluorescence of an absorbing solution or dispersion, it is important to correctly quantify the fluorescence intensity. Indeed, the absorbance of the dispersion impacts the intensity of the light reaching the centre of the cuvette as well as the amount of emitted light reaching the detector. Thus, when comparing the fluorescence of the silica layer, the fluorescence intensity must be corrected to consider the absorbance (ESI methods, Fig. S12 $\uparrow$ ). By correcting the fluorescence intensity of each dispersion for their respective absorbance, it is possible to see that the fluorescence of the silica matrix has the same intensity for all the samples and increases in line with the NP concentration (ESI Fig. S13†). Therefore, the presence of the iron oxide cores affects the overall brightness of the NP dispersions, with $\mathrm{Fe}_{3} \mathrm{O}_{4}$ having a larger effect than $\gamma-\mathrm{Fe}_{2} \mathrm{O}_{3}$. However, this effect can be explained by the excitation and emission of the fluorophore being in the same spectral region as the absorbance of the iron oxide cores and not as a result of any energy transfer process that the core may have on the fluorophore, as has been observed in other studies. ${ }^{45}$

The choice of a different fluorophore with excitation and emission at a different wavelength could prevent this issue. However, because FITC is widely used in biology due to its low cost and compatibility with the commonly used $488 \mathrm{~nm}$ laser, we considered it interesting for the scientific community to report a method to overcome the limitations related to the preparation of magnetic particles with this dye.

\section{Optimisation of protective layer}

Amorphous silica NPs, obtained via sol-gel processes such as the Stöber method, are known to dissolve at different rates when placed in biological media, dependent on $\mathrm{pH}$ and the presence of salts. During degradation, dye-doped NPs release the fluorophore with a consequent decrease in brightness and an increase in background noise. To protect silica NPs from fast dissolution, it is possible to use different strategies that lead to more resistant silica compositions ${ }^{46,47}$ such as the arginine-catalysed synthesis mentioned previously. ${ }^{39}$ However, the presence of amino acids on the NP surface may alter the composition of the biomolecular corona, which is formed when NPs encounter a biological media, and has been shown to be one of the critical factors determining NP uptake and intracellular trafficking. ${ }^{1}$

Here we report a new method of synthesizing a protective layer of silica adapted from Mahon et al. ${ }^{39}$ It consists of the thermo-catalysed hydrolysis and condensation of TEOS in the absence of any catalytic agent in solution.

Protective layers of different thicknesses were grown by adding different equivalents of TEOS (Fig. 4). The resulting thickness of the protective layer was in agreement with the theoretical value calculated as described in eqn (1) (see the Materials and methods section). 


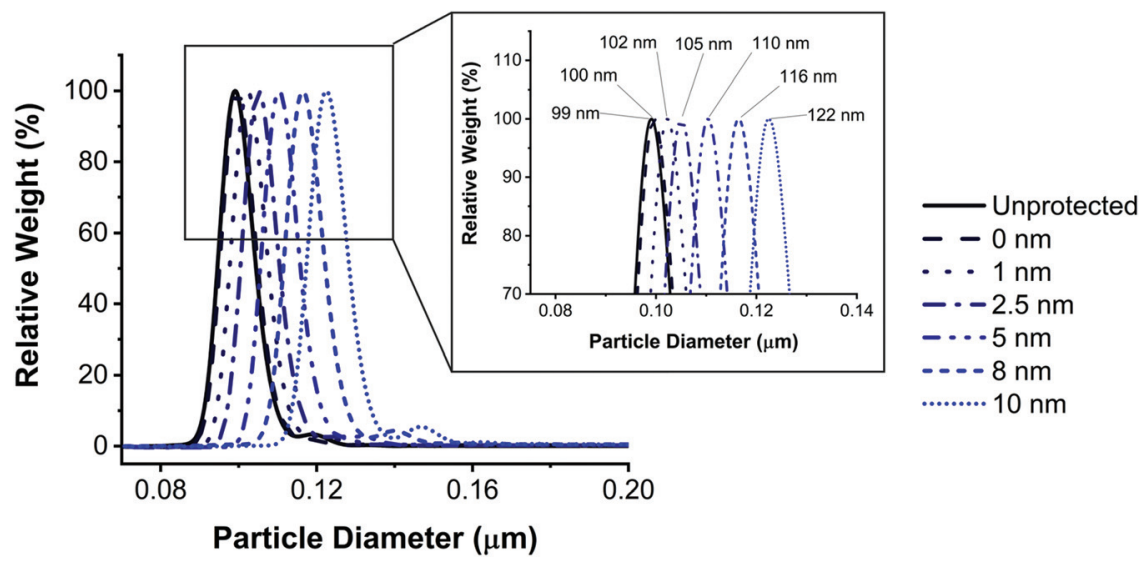

Fig. 4 Size characterization of protected FITC doped $\mathrm{SiO}_{2}$ NPs. DCS analysis of FITC doped $\mathrm{SiO}_{2}$ NPs protected using different volumes of TEOS and the inset shows a zoom of the peaks depicting their exact sizes. The thickness of the protective layer formed via the thermal-catalysed method is finely controlled.

The protective properties of the layers (of increasing thickness) were evaluated by incubating the NPs in cell culture media over a range of time periods $(0,4,8$ and $24 \mathrm{~h})$ at $37{ }^{\circ} \mathrm{C}$, then separating the leaked dye from the NPs using sodium dodecyl sulphate-polyacrylamide gel electrophoresis (SDS-PAGE; Fig. 5a). Additionally, some of the cell culture media in which the NPs were incubated was centrifuged to remove the NPs, and the residual fluorescence of the supernatant was measured and plotted against time of incubation (Fig. 5b).

A $1 \mathrm{~nm}$ layer prevented leaking for up to 4 hours incubation in biological media, while an $8 \mathrm{~nm}$ layer prevented leaking for up to 24 hours (Fig. 5). We hypothesise that, following this trend, a layer thicker than $8 \mathrm{~nm}$ could prevent leaking for a period even longer than 24 hours. A layer of silica of similar thickness grown under Stöber conditions does not provide the same level of protection against leaking in media (ESI Fig. S14 and $\mathrm{S} 15 \dagger$ ), as previously reported also by Mahon et al. ${ }^{39}$ The residual fluorescence of the NPs in the wells of the SDS-PAGE proves that despite leaking, some of the fluorescence is still detectable after 24 hours for all samples, except for the unprotected one.

Elemental microanalysis (CHN) and IR spectroscopy of the $\mathrm{SiO}_{2}$ NPs before and after protection (ESI methods, Table S3 and Fig. S16†) suggest that the high temperature catalyses the hydrolysis and condensation of TEOS and the removal of residual ethoxy groups from the silica matrix formed under Stöber conditions, thereby leading to the formation of a silica matrix more resistant to dissolution. Furthermore, in the absence of a catalytic agent to catalyse the hydrolysis and condensation of TEOS, it is only the temperature, the surface located acido-basic function $\mathrm{Si}-\mathrm{O}^{-}$, and its counter-ion ammonium that serves to catalyse the reaction. Moreover, as TEOS is immiscible with water, only a small amount is available in solution. For these reasons, we hypothesise that these experimental conditions promote the growth of a silica layer by the addition of monomers of hydrolysed or partially hydrolysed TEOS. This mechanism of silica formation, similar to that previously reported by Hristov et al. in $2015,{ }^{48}$ would differ significantly to the mechanism involved under the Stöber process, in which it is suggested that the silica matrix is formed by aggregation of nuclei leading to a nanoporous structure lacking condensation. ${ }^{49}$ However, further investigations are required to unravel the mechanism of the reaction occurring during the protection stage. A decrease of the NPs fluorescence after protection was also observed, mainly due to the degradation of the organic dye during the high temperature treatment (Fig. S17†).

For biological experiments, $\mathrm{Fe}_{3} \mathrm{O}_{4}$ NPs were chosen as multicores due to their higher saturation magnetisation compared to the maghemite and the fluorescent layer was protected with a $10 \mathrm{~nm}$ layer to prevent leaking for up to 24 hours in media. The final nanoconstructs $\left(\mathrm{Fe}_{3} \mathrm{O}_{4} @ \mathrm{SiO}_{2}\right.$ FITC-P) were characterized before use and consistency between different batches was preserved (Fig. S18-S20†). The mass magnetisation saturation of the final nanoconstruct $\left(13 \mathrm{Am}^{2} \mathrm{~kg}^{-1}\right)$ is lower than the bare $\mathrm{Fe}_{3} \mathrm{O}_{4}$ NPs $\left(80 \mathrm{Am}^{2} \mathrm{~kg}^{-1}, \mathrm{~S} 21 \dagger\right)$ which is in agreement with the fact that the diamagnetic $\mathrm{SiO}_{2}$ shell represents about $80 \%$ of the mass of the final particle.

\section{Nanoparticle performance in the biological environment}

While the particles were shown to not leak dye in cell culture media for up to 24 hours (Fig. 5 and S19c $\dagger$ ), the intracellular environment is more complex and constantly evolving, with changes in $\mathrm{pH}$, osmotic pressure and enzymatic activity, especially throughout the endo-lysosomal pathway (through which most NPs are trafficked). ${ }^{50}$ As such, it is important to ensure that the NPs are stable both during cell exposure (NPs in culture media) and after internalisation (NPs in the intracellular environment). Therefore, the optimised $\mathrm{Fe}_{3} \mathrm{O}_{4} @ \mathrm{SiO}_{2}$ FITC-P NPs were assessed for their suitability for in vitro intracellular imaging and flow cytometry analysis. 
a.

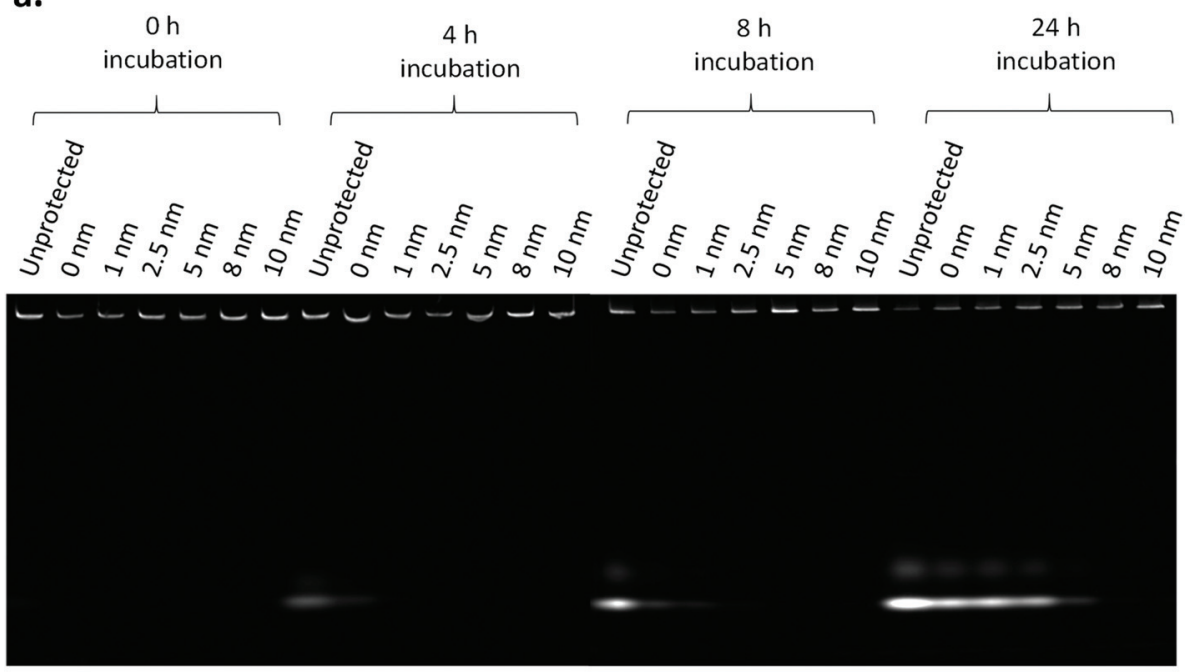

b.

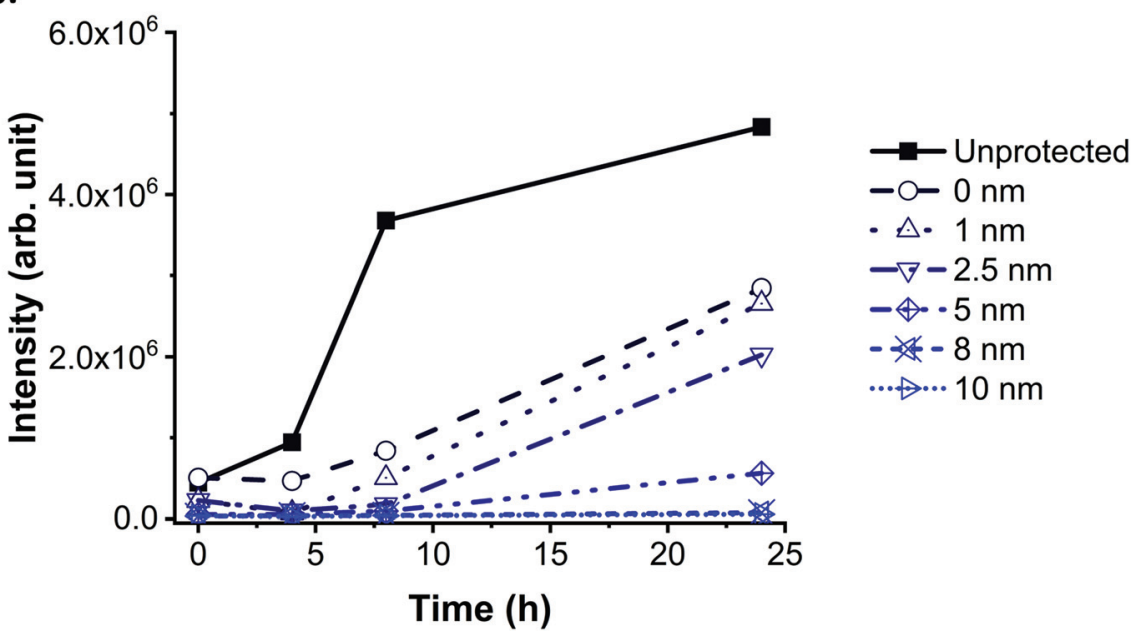

Fig. 5 Nanoparticle leaking test in biological media. (a) SDS-PAGE of FITC doped $\mathrm{SiO}_{2}$ NPs with protective layer of different thickness in media after incubation at $37^{\circ} \mathrm{C}$ for different time periods. $\mathrm{SiO}_{2} \mathrm{NPs}$ are too large to enter the gel and only free dye migrates through the gel. Therefore, the fluorescent bands on the bottom of the gel indicate the presence of free (and hence leaked) FITC. (b) Residual fluorescence in the media after FITC doped $\mathrm{SiO}_{2}$ NPs with protective layer of different thickness were spun down. The protective layer prevents leaking in biological conditions depending on the thickness, with a layer of $8-10 \mathrm{~nm}$ required for 24 hours stability. Note: the lines serve as a guide only and do not represent a fit.

The fluorescence of the NPs remains stable after 72 hours of cell incubation, and their intracellular location can be clearly identified by confocal microscopy (Fig. 6a). The absence of a green background or diffuse green signal, typical of cells treated with leaky NPs, ${ }^{51}$ demonstrates the stability of these particles in the dynamic intracellular environment.

Observing NP behavior in living cells is a key approach to understanding bio-nano interactions, as it allows the observation of intracellular NP dynamics while avoiding fixativerelated artefacts and alterations of the sample. NP uptake kinetics $^{52}$ and intracellular trajectories can also be investigated using live cell imaging. Multicolor live cell imaging can be used to perform time resolved co-localization studies to understand which compartments of the cell are involved in the response to NP treatment. ${ }^{53}$ The $\mathrm{Fe}_{3} \mathrm{O}_{4} @ \mathrm{SiO}_{2}$ FITC-P NPs were tested in live cell imaging experiments using two common conditions: long-term/low frequency (i.e. overnight time-lapses, a few frames per hour acquired), typically useful for observing slow cellular responses and trafficking pathways, and short-term/high frequency acquisition (i.e. a few minutes long, many frames per second acquired), typically used to record fast cellular dynamics. In both acquisition formats, the fluorescence intensity of the particles was stable throughout the analysis time (Fig. S22 and S23†) with NP internalisation and intracellular accumulation clearly observed (Fig. 6b).

Furthermore, these NPs were suitable for use in superresolution radial fluctuations (SRRF) imaging (Fig. 6c), making them ideal for super-resolved intracellular localisation analysis 
a.

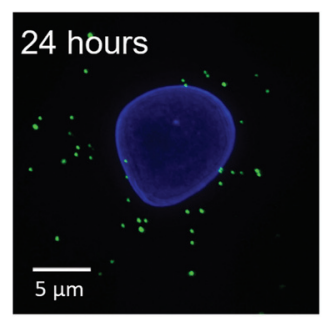

b. I
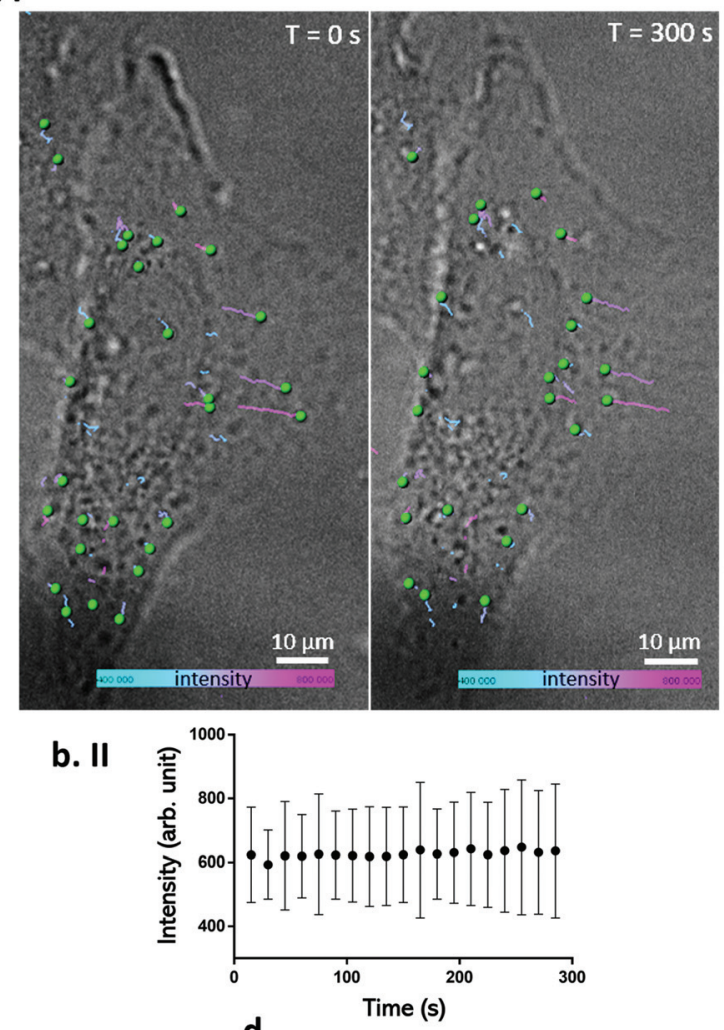

d. c.

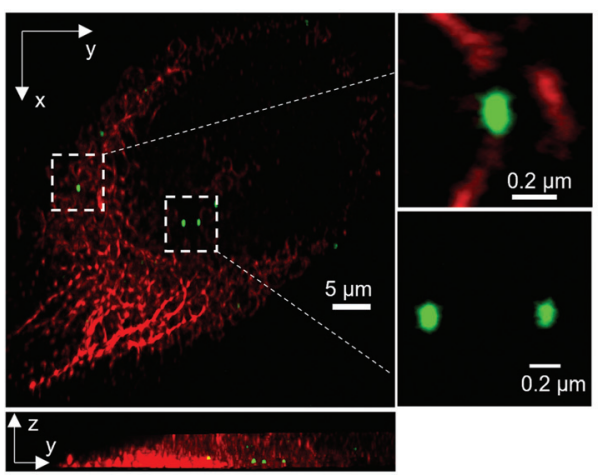

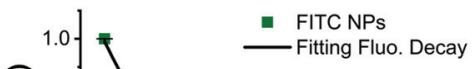

Fitting Fluo. Decay

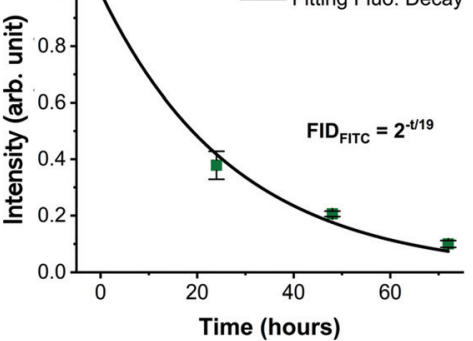

Fig. 6 Image analysis of the cell internalized $\mathrm{Fe}_{3} \mathrm{O}_{4} \mathrm{aSiO}_{2} \mathrm{FITC}-\mathrm{P}$ NPs. (a) Confocal images of fixed A549 cells treated with $40 \mu \mathrm{mL} \mathrm{m}^{-1}$ of $\mathrm{Fe}_{3} \mathrm{O}_{4} @ \mathrm{QSiO}_{2}$ FITC-P for 2 hours and incubated in fresh culture media for 24,48 or 72 hours. The nanoparticles are detectable in the intracellular environment as bright punctuated green spots. Blue: cell nuclei, green: $\mathrm{Fe}_{3} \mathrm{O}_{4} \mathrm{aSiO}_{2}$ FITC-P. Scale bar: $5 \mu$ m. (b. I) Live cell imaging time-lapse showing NPs intracellular trafficking after exposing the cells to $40 \mu \mathrm{g} \mathrm{mL}^{-1}$ for 2 hours. The NPs motion was tracked from $T=0 \mathrm{~s}$ (left panel, first frame of the movie) where the NPs are at the beginning of their route, until $T=300 \mathrm{~s}$ (right panel, last frame of the movie) where the NPs are at the end of their trajectory (see ESI movie $1 \uparrow$ for the real time trajectory generation). The tracked NPs are rendered as green spheres, and their trajectories are presented as color coded lines, representing the single particle fluorescence intensity variation overtime. The minimum intensity (400 arb. unit) is represented in turquoise, the maximum intensity (800 arb. unit) is in pink. A variation in the trajectory color corresponds to a NP FITC intensity variation. Acquisition frequency: 0.06 frames per s. Grey scale: bright field; green: $\mathrm{Fe}_{3} \mathrm{O}_{4}\left(\mathrm{CSiO}_{2} \mathrm{FITC}-\mathrm{P}\right.$, scale bar: $5 \mu$ m. (b. II) The mean NP fluorescence intensity remained stable over the entire time-lapse acquisition length (values are expressed as mean \pm SD). (c) 3D SRRF super resolution image of $\mathrm{A} 549$ cells treated with $40 \mu \mathrm{g} \mathrm{mL} \mathrm{L}^{-1}$ of $\mathrm{Fe}_{3} \mathrm{O}_{4} \mathrm{QSSO}_{2}$ FITC-P. The NPs had adequate emission intensity and photostability to sustain SRRF super resolution imaging. Red: tubulin, green: NPs. Scale bar: $5 \mu \mathrm{m}$. (d) Flow cytometry analysis of A549 cells treated with $80 \mu \mathrm{g} \mathrm{mL}{ }^{-1}$ of $\mathrm{Fe}_{3} \mathrm{O}_{4} \mathrm{aSiO}_{2}$ FITC-P NPs for 2 hours and cultured in NP-free media for different timepoints. The fluorescence signal from NPs follows a base two exponential decay fitting with a $R^{2}=0.99$. The half-life of the FITC signal is in close agreement with the A549 division time, confirming leaking is absent or negligible (values are expressed as mean $\pm \mathrm{SD}, n=2$ ).

in fixed or live cells, which is a key aspect of drug delivery studies. ${ }^{54}$ The main drawback associated with super-resolution techniques such as stimulated emission depletion (STED) and stochastic optical reconstruction microscopy (STORM), is the inability to perform live cell analysis. With these methods, the targets are precisely localised based on the intermitting emis- 
sion of the fluorophore, induced by stimuli such as high intensity laser exposure or reducing buffers that strongly affect live cell viability. ${ }^{55,56}$ Furthermore, acquisition using these techniques is extremely time consuming, not all the commercially available fluorophores are compatible, and they are easily photobleached under the required acquisition conditions. ${ }^{57}$ Contrary to other super-resolution techniques, SRRF does not require a blinking fluorophore to achieve a resolution in the order of $30-50 \mathrm{~nm},{ }^{58}$ instead, it is based on a post-acquisition image analysis of an image sequence composed of hundreds of frames. The algorithm defines the pixel signal as real (generated by a fluorophore) or as not real (background noise) based on the radial symmetry of pixels across the image sequence. The symmetry is greater for fluorophores, as the emission is precisely localized among the frames, and smaller for background noise which is randomly distributed across the image sequence. ${ }^{59}$ The superresolved final image is then reconstructed based on the radial symmetry analysis that calculates the original position of the fluorophores and excludes noise, overcoming the Abbe diffraction limit.

Fluorophores employed in SRRF imaging must have adequate emission intensity and photostability to sustain exposure acquisition settings in the order of a few milliseconds and capture in hundreds of sequential frames. ${ }^{59}$ If these conditions are not met, subsequent image reconstruction can lead to artefacts or failure. ${ }^{54}$ The $\mathrm{Fe}_{3} \mathrm{O}_{4} @ \mathrm{SiO}_{2}$ FITC-P NPs were bright with a punctuated signal and size in the expected range (ca. $0.1 \mu \mathrm{m}$ ) when imaged using SRRF super resolution microscopy. They could be easily seen interacting with tubulin (red filaments, top inset Fig. 6c), a main component of the cell cytoskeleton that plays a role in the movement of vesicles through the endo-lysosomal pathway (which most NPs are processed through). The ability to precisely identify the location of NPs during their interaction with cells is critically important for overcoming the main limitations in nanomedicine, and the use of these $\mathrm{Fe}_{3} \mathrm{O}_{4} @ \mathrm{SiO}_{2}$ FITC-P NPs in conjunction with SRRF microscopy provides adequate resolution for in-depth intracellular particle trafficking studies.

The intracellular stability of the NPs was also confirmed by flow cytometry analysis (Fig. 6d and Fig. S23†). The FITC intensity per cell was monitored for up to 72 hours and the signal decreased in an exponential fashion, with a halving time of the FITC signal (about 19 hours, see ESI $\dagger$ methods) close to the division time of the cell line (dividing cells will split their NP load, resulting in a reduction in the FITC signal per cell that is unrelated to dye leaking). ${ }^{60}$ Cells treated with leaking NPs typically show a significant loss of fluorescence at a greater rate than proliferation. ${ }^{60}$ Thus, the flow cytometry analysis supports the determination that the fluorescence of these particles is stable intracellularly up to 72 hours.

In addition to the effective fluorescent properties of these NPs, the magnetic multicore offers the advantage of easy extraction from complex biological solutions, providing the opportunity for analysis of many aspects of the NP interactions with the biological machinery.

To demonstrate that the NPs can be harvested after cell internalisation, A549 cells were incubated with the $\mathrm{Fe}_{3} \mathrm{O}_{4} @ \mathrm{SiO}_{2}$ FITC-P NPs for 24 hours, after which the cells were collected and lysed. The NPs were then recovered from a.

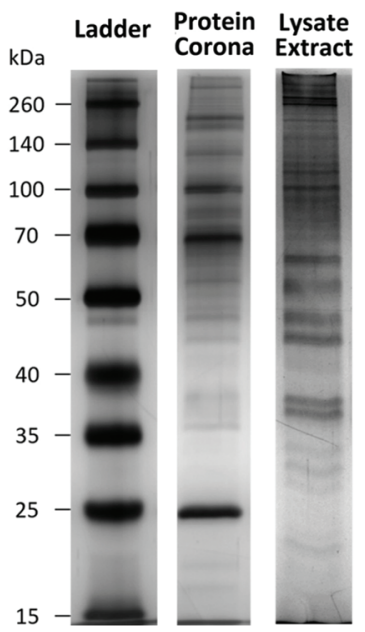

b.
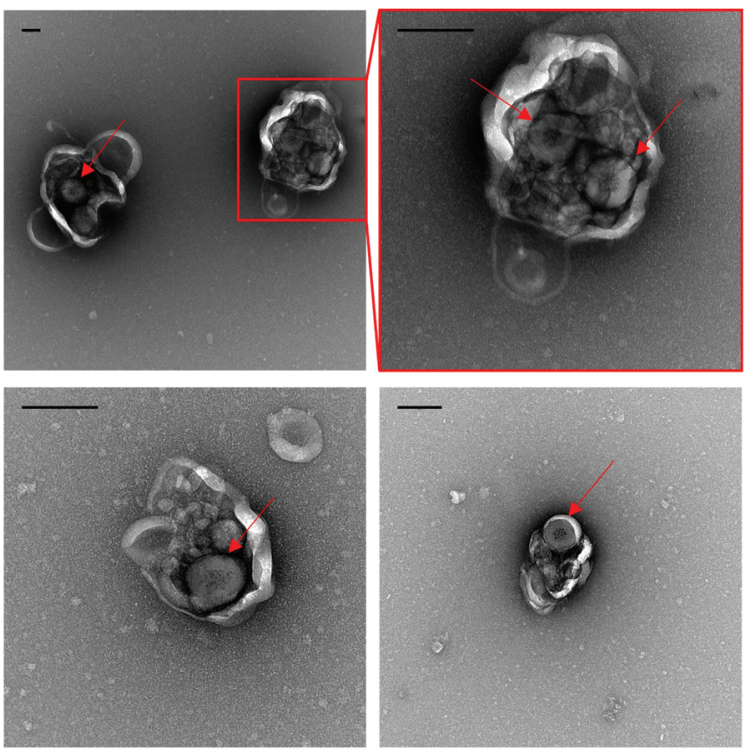

Fig. 7 Analysis of $\mathrm{Fe}_{3} \mathrm{O}_{4} \mathrm{QSSiO}_{2}$ FITC-P NPs after cell internalization. (a) Comparison of protein composition of $\mathrm{Fe}_{3} \mathrm{O}_{4} @ \mathrm{CSiO}_{2} \mathrm{FITC}^{-\mathrm{P}}$ protein corona before and after incubation in A549 cells by silver-stained SDS-PAGE gel. The protein profile of the NPs extracted from the intracellular environment is significantly different, indicating they have retained proteins from their intracellular environment. (b) TEM imaging of extracted NPs reveals they are contained within vesicle like structures (scale bar: $200 \mathrm{~nm}$ ). Arrows were added to help the reader to identify the particles. 
the lysate by magnetic extraction. The protein profile associated with the cell extracted particles is significantly different to that of the simple $\mathrm{Fe}_{3} \mathrm{O}_{4} @ \mathrm{SiO}_{2}$ FITC-P NPs with hard corona (pre-formed in complete medium (cMEM)) before cell incubation (see Materials and methods for further information; Fig. 7a). Interestingly, the protein content per NP in the extracted sample is much higher than that of the pre-formed biomolecular corona (ESI Fig. S24†). Particles and biological structures were identified in the magnetically extracted population by TEM analysis (ESI Fig. S25 $\dagger$ ). Of particular interest was the observation that the NPs were contained within intact vesicle-like structures (Fig. 7b). This finding demonstrates the potential to harvest internalised particles from the intracellular environment while successfully preserving the biological structures in which they are encapsulated and highlights their potential use as a tool to analyse the processes that regulate intracellular trafficking. ${ }^{2}$

\section{Conclusions}

Investigating the interactions of NPs with cells usually relies on a combination of techniques such as fluorescence microscopy, flow cytometry and proteomics. However, the cross-interpretation of the results can be challenging due to the lack of correlation when different NP systems are required. Fluorescent microscopy is one of the most powerful techniques for investigating intracellular organization as it allows NPs to be imaged after internalisation in cells. However, fluorescent microscopy relies on the labelling of specific biomolecules and so has limited capacity for identifying the interaction partners of NPs. Magnetic NPs can be easily extracted from a biological media by magnetic extraction, and the interacting biomolecules can be co-extracted with the NPs and identified. Multifunctional NPs may provide a solution to these challenges.

In this study, we presented the design and optimisation of multifunctional magnetic and fluorescent NPs that are suitable for the most common imaging techniques and also the extraction of cell compartments involved in their intracellular trafficking. The NP fluorescence intensity is derived from a fluorescein-based dye covalently encapsulated in a silica layer on the particle. The brightness and stability of these NPs was shown to be compatible with imaging techniques such as confocal microscopy, 3D SRRF imaging and live cell imaging. The particles were designed to minimise silica degradation in biological environments which would induce the loss of the dye during live imaging. The fluorescence of the particles was easily detected in the cell after $72 \mathrm{~h}$, demonstrating their ability to be tracked over extended time periods. We also showed that, owing to the magnetic multicore, the internalized NPs could be extracted from the cell after lysis enabling the analysis of the proteins involved in their intracellular trafficking. This new construct could open the way to the correlation of the intracellular location of the NPs with the identification of the biomolecules interacting with them.

\section{Materials and methods}

\section{Synthesis of $\mathrm{SiO}_{2}$ cores}

The synthesis of the $\mathrm{SiO}_{2}$ NPs was based on a two-step seedgrowth process adapted from a synthesis already reported. ${ }^{61}$ Briefly, a solution made of $21.6 \mathrm{~mL}$ of ethanol and $240 \mu \mathrm{L}$ of TEOS (Sigma - 131903) was added quickly to a solution composed of $21.6 \mathrm{~mL}$ of ethanol, $114 \mu \mathrm{L}$ of MilliQ water, and $1.18 \mathrm{~mL}$ of $\mathrm{NH}_{3} 35 \%$ (Fisher - 10659263). The mixture was allowed to react at room temperature for 12 hours, resulting in $\mathrm{SiO}_{2}$ NPs of about $15 \mathrm{~nm}$ in diameter. Seven millilitres of the resulting dispersion were added in a solution containing $57.8 \mathrm{~mL}$ of ethanol, $19.6 \mathrm{~mL}$ of MilliQ water and $1.12 \mathrm{~mL}$ of ammonia solution $35 \%$. TEOS was added in $100 \mu \mathrm{L}$ aliquots to the reaction solution and the growth of the NPs was monitored by dynamic light scattering (DLS) until the size of $86 \mathrm{~nm}$ was obtained.

\section{Synthesis of $\mathrm{Fe}_{3} \mathrm{O}_{4} \mathrm{NPS}$}

Magnetite NPs were synthesized by a coprecipitation method. ${ }^{62,63}$ In a typical synthesis, $0.030 \mathrm{~mol}$ of $\mathrm{FeCl}_{3} \cdot 6 \mathrm{H}_{2} \mathrm{O}$ (Sigma - 31232) were dissolved in $350 \mathrm{~mL}$ of milliQ water, while $0.015 \mathrm{~mol}$ of $\mathrm{FeCl}_{2} \cdot 4 \mathrm{H}_{2} \mathrm{O}$ (Sigma - 44939) were dissolved in $17 \mathrm{~mL}$ of $\mathrm{HCl} 1.5 \mathrm{M}$. The two iron solutions were combined under vigorous mechanical stirring resulting in a light-yellow solution. While stirring, $30 \mathrm{~mL}$ of ammonia solution $35 \%$ were added to the iron solution and the formation of iron oxide NPs was immediately observed.

After 15 minutes NPs were precipitated by magnetic decantation and washed 3 times with water before being redispersed in a slightly acidic aqueous solution of hydrochloric acid.

\section{Synthesis of $\gamma-\mathrm{Fe}_{2} \mathrm{O}_{3} \mathrm{NPs}$}

The synthesis of the $\gamma-\mathrm{Fe}_{2} \mathrm{O}_{3}$ NPs was carried out as reported by Mornet et al. ${ }^{63}$ Briefly, $\mathrm{Fe}_{3} \mathrm{O}_{4}$ nanoparticles were synthesised as described above but after washing three times in water, $50 \mathrm{~mL}$ of $\mathrm{HNO}_{3} 2 \mathrm{M}$ was added and the particles were stirred for 15 minutes. NPs were precipitated and redispersed in $60 \mathrm{~mL}$ of $\mathrm{Fe}(\mathrm{III})$ nitrate $0.3 \mathrm{M}$ and boiled for 30 minutes; a colour change from black to rust brown was observed. The solution was allowed to cool at room temperature before the NPs were precipitated by magnetic decantation. After removal of the supernatant, $50 \mathrm{~mL}$ of $\mathrm{HNO}_{3} 2 \mathrm{M}$ was added and the particles were stirred for 15 minutes. Then, NPs were precipitated by magnetic decantation and the pellet washed 4 times with acetone before being redispersed in MilliQ water. Trace acetone was removed by rotary evaporator.

\section{Synthesis of iron oxide multicores}

The synthesis of the iron oxide multicores and their encapsulation in a silica matrix was adapted from a procedure described by Yammine et $a l .{ }^{40}$ Briefly, for the synthesis of multicores, iron oxide NPs were transferred in cyclohexane, by coating them with oleic acid according to the procedure described by Van Ewijk et al. ${ }^{64}$ Five millilitres of the hydrophobic ferrofluid at $50 \mathrm{mg} \mathrm{mL}^{-1}$ were mixed with a solution of SDS $50 \mathrm{mM}$ and 
emulsified in a $25 \mathrm{~mL}$ round bottom flask, with a tip sonicator (Vibra-cell $^{\mathrm{TM}}$ VCX 750) (time: $20 \mathrm{~min}$; amplitude: $40 \%$; pulse: 2 $\mathrm{s}$ on, $3 \mathrm{~s}$ off) in an ice bath. The emulsion was then transferred in a $100 \mathrm{~mL}$ round bottom flask and heated to $80{ }^{\circ} \mathrm{C}$ with a soft flow of air for 90 minutes, to evaporate the cyclohexane (water was added when necessary to prevent drying of the sample). The dispersion was then diluted with MilliQ water to reach a total volume of $50 \mathrm{~mL}$ and heated to $40{ }^{\circ} \mathrm{C}$. Under stirring, $2.5 \mathrm{~mL}$ of ammonia solution (35\%) were added together with $250 \mu \mathrm{L}$ of TEOS. After 2 hours the dispersion was diluted by addition of $200 \mathrm{~mL}$ of water and precipitated by centrifugation. The supernatant was discarded, and the pellet redispersed in water $(20 \mathrm{~mL})$.

A basic hydroalcoholic solution of $72 \mathrm{~mL}$ of ethanol, $4.4 \mathrm{~mL}$ of ammonia solution $35 \%$ and $100 \mu \mathrm{L}$ of TEOS was prepared and the particles dispersed in water were added to this solution under stirring. After 30 minutes, a second aliquot of $200 \mu \mathrm{L}$ of TEOS was added followed by another aliquot of $300 \mu \mathrm{L}$ after other 30 minutes. The reaction was left for 2 hours, before the ammonia was evaporated by rotavapor and NPs precipitated by centrifugation. The pellet was washed three times and redispersed in $20 \mathrm{~mL}$ of milliQ water. Aggregates were spun down at $600 \mathrm{~g}$ for 10 minutes.

\section{Dye solution preparation}

Fluorescein isothiocyanate (Sigma - F7250). When not otherwise stated, $4 \mathrm{mg}$ of FITC $(10 \mu \mathrm{mol})$ were dissolved in $1 \mathrm{~mL}$ of ethanol. Then, $18 \mu \mathrm{L}$ of APTMS (Sigma - 281778) $(100 \mu \mathrm{mol})$ were added and it was noted the solution turned bright orange. The solution was allowed to react for $12 \mathrm{~h}$ at $4{ }^{\circ} \mathrm{C}$ and was then used within one day for the $\mathrm{SiO}_{2} \mathrm{NP}$ synthesis.

Rhodamine B isothiocyanate (Sigma - 283924). To $0.5 \mathrm{~mL}$ of a solution of RB $2 \mu \mathrm{mol} \mathrm{mL} \mathrm{mL}^{-1}$ were added $10 \mu \mathrm{mol}$ of APTMS. The reaction was allowed to react for $4 \mathrm{~h}$ at room temperature before use.

Fluorescein-5-maleimide (TCI - F0810). To $0.5 \mathrm{~mL}$ of a solution of FM $2 \mu \mathrm{mol} \mathrm{mL} \mathrm{m}^{-1}$ were added $4 \mu \mathrm{mol}$ of MPTMS (Sigma - 175617). The reaction was allowed to react for $4 \mathrm{~h}$ at room temperature before use.

Cyanine3 - maleimide (Lumiprobe - 51080). Cy3-maleimide was solubilized in DMSO at a concentration of $50 \mathrm{~g} \mathrm{~L}^{-1}$ and stored at $-20{ }^{\circ} \mathrm{C}$ before use. Cy3-maleimide was diluted in $0.5 \mathrm{~mL}$ of EtOH to a final concentration of $2 \mu \mathrm{mol} \mathrm{mL} \mathrm{m}^{-1}$ and $4 \mu \mathrm{mol}$ of MPTMS were added. The reaction was allowed to react for $4 \mathrm{~h}$ at room temperature before use.

Cyanine5 - maleimide (Lumiprobe - 53080). Cy5-maleimide was solubilized in DMSO at a concentration of $50 \mathrm{~g} \mathrm{~L}^{-1}$ and stored at $-20{ }^{\circ} \mathrm{C}$ before use. Cy5-maleimide was diluted in $0.5 \mathrm{~mL}$ of EtOH to a final concentration of $2 \mu \mathrm{mol} \mathrm{mL} \mathrm{m}^{-1}$ and $4 \mu \mathrm{mol}$ of MPTMS were added. The reaction was allowed to react for $4 \mathrm{~h}$ at room temperature before use.

Cyanine7 - maleimide (Lumiprobe - 55080). Cy7-maleimide was solubilized in DMSO at a concentration of $50 \mathrm{~g} \mathrm{~L}^{-1}$ and stored at $-20{ }^{\circ} \mathrm{C}$ before use. Cy7-maleimide was diluted in $0.5 \mathrm{~mL}$ of EtOH to a final concentration of $2 \mu \mathrm{mol} \mathrm{mL} \mathrm{m}^{-1}$ and
$4 \mu \mathrm{mol}$ of MPTMS were added. The reaction was allowed to react for $4 \mathrm{~h}$ at room temperature before use.

\section{Synthesis of fluorescent shell for $\mathrm{SiO}_{2}$ cores}

For the synthesis of the fluorescent shell, $2 \mathrm{~mL}$ of $\mathrm{SiO}_{2}$ cores in the growing solution were transferred in a reaction tube. An aliquot of $20 \mu \mathrm{L}$ of TEOS was added followed by a $16 \mu \mathrm{L}$ aliquot of dye solution (this volume provides an incorporation of APTMS-FITC of $0.5 \%$, in the course of the dye loading study, this volume was adjusted to give the desired percentage). After 30 minutes, a second aliquot of $40 \mu \mathrm{L}$ of TEOS and $32 \mu \mathrm{L}$ of dye solution was added to the reaction solution followed, after another 30 minutes, by a third aliquot of $40 \mu \mathrm{L}$ of TEOS and $32 \mu \mathrm{L}$ of dye solution. The reaction was considered finished 2 hours after the last addition of TEOS, at room temperature.

The reaction solution was diluted 1 in 5 in milliQ water before being precipitated by centrifugation. The pellet was washed 3 times with water and then redispersed in water.

\section{Synthesis of fluorescent shell for magnetic multicores}

For the synthesis of the fluorescent shell on iron oxide multicores, $7 \mathrm{~mL}$ of a dispersion $7 \mathrm{mg} \mathrm{mL}^{-1}$ of multicores were added in a reaction solution made of $25.7 \mathrm{~mL}$ ethanol and $1.6 \mathrm{~mL}$ of ammonia solution $35 \%$. An aliquot of $100 \mu \mathrm{L}$ of TEOS was added at the same time with $80 \mu \mathrm{L}$ of dye solution. After 30 minutes, a second aliquot of $200 \mu \mathrm{L}$ of TEOS and $160 \mu \mathrm{L}$ of dye solution were added and after a further 30 minutes a third aliquot of $200 \mu \mathrm{L}$ of TEOS and $160 \mu \mathrm{L}$ of dye solution were added. The reaction was left for 2 hours at room temperature until completion. The reaction solution was diluted 1 in 5 in milliQ water before being precipitated by centrifugation. The pellet was washed 3 times with water and then was redispersed in water. Potential aggregates were removed by centrifuging at $600 \mathrm{~g}$ for 10 minutes.

\section{Synthesis of the protective layer}

A dispersion of $\mathrm{SiO}_{2} \mathrm{NPs} 20 \mathrm{mg} \mathrm{mL}{ }^{-1}$ was heated to $90{ }^{\circ} \mathrm{C}$ and an aliquot of TEOS added. The volume of TEOS to be added can be calculated by the following formula:

$$
V_{\mathrm{TEOS}}=\frac{4 \times \mathrm{MW}_{\mathrm{TEOS}} \times d_{\mathrm{SiO}_{2}} \times \pi \times\left(R^{3}-r^{3}\right) \times N_{\mathrm{NPS}}}{3 \times d_{\mathrm{TEOS}} \times \mathrm{MW}_{\mathrm{SiO}_{2}}}
$$

where we consider $d_{\mathrm{SiO}_{2}}=2 \mathrm{~g} \mathrm{~cm}^{-3}$ (dependent on their size and synthesis method $\left.{ }^{29,65}\right)$. $N_{\mathrm{NPs}}$ is the number of NPs in the dispersion; $R$ is the final desired radius; and $r$ is the starting radius of the NPs in the dispersion.

A first aliquot equal to half of the total volume was added to the dispersion while heating to $90{ }^{\circ} \mathrm{C}$ and a second aliquot added after 1 hour. The reaction was left for 3 hours at $90{ }^{\circ} \mathrm{C}$ until completion. The NPs were then allowed to cool to room temperature, and then were spun down by centrifugation and washed three times with distilled water, before being redispersed in water. 


\section{Leaking test}

NPs were incubated in minimum essential medium (MEM) supplemented with 10\% foetal bovine serum (FBS, Gibco), 1\% penicillin-streptomycin (Invitrogen) at a concentration of $0.1 \mathrm{mg} \mathrm{mL} \mathrm{m}^{-1}$ at $37^{\circ} \mathrm{C}$ for $0,4,8$ and 24 hours. $20 \mu \mathrm{L}$ of such solution were then mixed with a $10 \times$ SDS loading buffer (New England Biolabs) and loaded in a gel $10 \%$ polyacrylamide. The gel was run at $200 \mathrm{mV}$ for 30 minutes and imaging performed with Syngene G-Box XT4 equipped with a $525 \mathrm{~nm}$ filter and a blue light.

\section{Cell culture}

The A549 non-small lung carcinoma (ATCC® CCL-185 ${ }^{\mathrm{TM}}$ ) cell line was purchased from ATCC, and cultured in MEM $\left(\mathrm{Gibco}^{\mathrm{TM}}\right)$ supplemented with $10 \% \mathrm{FBS}\left(\mathrm{Gibco}^{\mathrm{TM}}\right)$ and $50 \mathrm{U} \mathrm{mL}^{-1}$ penicillin-streptomycin (Gibco $\left.{ }^{\mathrm{TM}}\right)$. Cells were grown at $37^{\circ} \mathrm{C}$ in a humidified atmosphere of $5 \% \mathrm{CO}_{2}$ and sub-cultured at $70-80 \%$ confluence using trypsin $\left(0.25 \%\right.$ in EDTA; Gibco $\left.{ }^{\mathrm{TM}}\right)$. Cells were screened monthly for mycoplasma contamination using the MycoAlert ${ }^{\mathrm{TM}}$ Mycoplasma Detection Kit, and all cultures were free of contamination for the duration of experiments reported.

\section{$\mathrm{Fe}_{3} \mathrm{O}_{4} @ \mathrm{SiO}_{2}$ FITC-P NPs corona preparation}

$\mathrm{Fe}_{3} \mathrm{O}_{4} @ \mathrm{SiO}_{2}$ FITC-P NPs were incubated in cMEM (MEM supplemented with 10\% FBS (Gibco) and 1\% penicillin-streptomycin, Invitrogen) at a concentration of $0.8 \mathrm{mg} \mathrm{mL}^{-1}$ at $37^{\circ} \mathrm{C}$ for 1 hour. For the SDS-PAGE experiments, the NPs in cMEM were precipitated by centrifugation and redispersed in phosphate-buffered saline (PBS) and then washed twice with PBS by precipitation for magnetic decantation.

\section{Exposure of cells to NPs}

Cells were seeded at a density of $6 \times 10^{6}$ cells per flask in T175 flasks (Grenier) for extraction experiments. After seeding, cells were incubated for 48 hours before exposure to the NP dispersion. $\mathrm{Fe}_{3} \mathrm{O}_{4} @ \mathrm{SiO}_{2}$ FITC-P NPs with in situ corona were incubated at a concentration of $0.8 \mathrm{mg} \mathrm{mL} \mathrm{m}^{-1}$ with the cells for 10 minutes, then the non-internalized NPs were discarded and after two washes with Dulbecco's phosphate-buffered saline (DPBS) and two washes in cMEM. The cells were then incubated with fresh cMEM (without NPs) for a further 8-24 hours.

\section{Spinning disk confocal imaging}

Cells were imaged in a 96-well glass bottom plates (CellVis) on a Nikon eclipse TI spinning disc confocal microscope. For the samples shown in Fig. 6a and c, the cells were fixed with $4 \%$ paraformaldehyde and stained with DAPI $\left(2 \mu \mathrm{g} \mathrm{mL}{ }^{-1}\right.$, Fig. 6a) to visualise the cell nucleus; or permeabilised with $1 \% \mathrm{w} / \mathrm{w}$ saponin and immunostained with anti-tubulin antibody (Thermo Fisher, A11126) in combination with AlexaFluor-546 secondary antibody (Thermo Fisher, A11035). For live cells imaging shown in Fig. $6 \mathrm{~b}$ and ESI Fig. S22, $\uparrow$ the cells were imaged in phenol-free CMEM, in a live cell chamber at $37{ }^{\circ} \mathrm{C}$ with $5 \% \mathrm{CO}_{2}$. The images and the time-lapses were analyzed with Imaris imaging software (Bitplane).

\section{Nanoparticle uptake assessment by flow cytometry}

To prepare the cells for flow cytometry analysis, 50000 cells per well were seeded in a 24-well plate. After incubation with the nanoparticles, the cells were harvested by trypsinisation and washed twice in PBS. The cells were then fixed in $4 \%$ paraformaldehyde and stored in PBS at $4{ }^{\circ} \mathrm{C}$. To assess the NP dilution, the cells were run in a Cytoflex LX (Beckman Coulter) flow cytometer. The fluorescent signal per cell was measured (excitation/emission 488/530 nm for NPs) for $\geq 50000$ events collected in the P2 population (single cells gate, gating shown in ESI Fig. S23†).

\section{Cell lysis}

The cell culture media was discarded, and the cells were washed with $10 \mathrm{~mL}$ of PBS per flask. The PBS was discarded, and the cells were incubated with $5 \mathrm{~mL}$ per flask of trypsin at $37{ }^{\circ} \mathrm{C}$ for 10 minutes. The trypsin was deactivated by adding $5 \mathrm{~mL}$ per flask of cMEM supplemented with $10 \%$ FBS. The cell dispersion was transferred in a Falcon tube and cells were precipitated by centrifugation and washed once with PBS. $15 \times 10^{6}$ cells were redispersed in $4.5 \mathrm{~mL}$ of lysis buffer $(0.01 \times$ potassium phosphate-buffered saline (KPBS) + protease inhibitor) and allowed to swell for 10 minutes in ice. Cells were loaded in a Dounce homogenizer and lysed with 12 consecutive hits. The lysis buffer was neutralized adding $500 \mu \mathrm{L}$ of $10 \times$ KPBS.

\section{SDS-PAGE}

For SDS-PAGE, the lysate was extracted in a three-pole magnet for 8 hours at $4{ }^{\circ} \mathrm{C}$, the supernatant was discarded, and the pellet redispersed in PBS. The pellet was extracted once more for 12 hours at $4{ }^{\circ} \mathrm{C}$ and redispersed in PBS. The concentration of NPs in the extracted lysate was estimated by fluorescence measurement by interpolation on a calibration line.

$\mathrm{Fe}_{3} \mathrm{O}_{4} @ \mathrm{SiO}_{2}$ FITC-P NPs with hard protein corona and those extracted from the cell lysate were boiled for 10 minutes in the presence of 1,4-dithiothreitol (DTT, New England Biolabs) as a reducing agent. The samples were loaded in a $10 \%$ polyacrylamide gel and gel electrophoresis was performed at $110 \mathrm{mV}$ for 75 minutes. To detect the separated proteins, the gels were stained using the Pierce ${ }^{\mathrm{TM}}$ Silver Stain Kit. Imaging was performed with Syngene G-Box XT4 equipped with a UV filter and a white light source.

\section{TEM Negative staining}

For TEM imaging, the cell lysate was processed with MS magnetic columns (Miltenyi Biotec). The magnetic fraction of the cell lysate was absorbed onto hydrophilized (PELCO easiGlow $^{\mathrm{TM}}$ ) 200-mesh carbon/Formar coated Copper grids (TedPella, 01800). The samples were then further fixed with 2.5 wt $\%$ glutaraldehyde (Sigma-Aldrich, G7651) and $2 \%$ formaldehyde (Thermo Fisher Scientific, 28906) in 0.1 M sodium phosphate buffer, $\mathrm{pH}=7.4$ and negatively stained with a solution of 1 wt\% uranyl acetate (Agar Scientific, R1260A) and 
$0.1 \mathrm{wt} \%$. Trehalose (Sigma - Aldrich, T9449). The addition of $0.1 \mathrm{wt} \%$ trehalose to the staining solution aimed to improve the preservation of biological material during dehydration. The grids were left to air dry and representative TEM micrographs were obtained with FEI Tecnai G2 20 Twin operating at $200 \mathrm{kV}$.

\section{Rights retention statement}

This publication has emanated from research supported in part by grants from Science Foundation Ireland under Grant numbers 17/NSFC/4898, 17/ERCD/4962 and 15/SIRG/3423. For the purpose of Open Access, the author has applied a CC BY public copyright licence to any Author Accepted Manuscript version arising from this submission.

\section{Author contributions}

Lorenzo Cursi: conceptualization, data curation (NPs synthesis, characterization, biological application), writing-original draft preparation, investigation, methodology; Silvia Vercellino: data curation (optical imaging), investigation, methodology, writing-reviewing and editing; Mura McCafferty: conceptualization, investigation, data curation (flow cytometry), writing-reviewing and editing; Emily Sheridan: data curation (TEM imaging), writing-reviewing and editing, investigation; Vanya Petseva: methodology (TEM imaging), writingreviewing and editing, investigation; Laurent Adumeau: conceptualization, supervision, writing-reviewing and editing; Kenneth A. Dawson: supervision, conceptualization, writingreviewing and editing.

\section{Conflicts of interest}

There are no conflicts to declare.

\section{Acknowledgements}

KAD, LA, ES and SV acknowledge that this publication has emanated from research supported in part by grants from Science Foundation Ireland [17/NSFC/4898 (KAD, LA and ES), 17/ERCD/4962 (KAD) and 15/SIRG/3423 (SV)]. LC and VP acknowledge this work was part-funded by the Celtic Advanced Life Science Innovation Network (CALIN), an Ireland-Wales INTERREG project part-funded by the European Regional Development Fund through the Welsh Government, agreement no.80885. SV also acknowledges support by the PhD Research Scholarship from the UCD School of Biomolecular and Biomedical Science. MMC acknowledges the support of the Irish Research Council Enterprise Partnership Scheme (Postdoctoral) 2019 - EPSPD/ 2019/232. The authors acknowledge Dr. D. Scholtz and Dr. A. Blanco from the imaging and flow cytometry core facili- ties of the Conway Institute of Biomolecular and Biomedical Research Centre at University College Dublin for their assistance. The authors acknowledge Delyan Hristov and Eugene Mahon for their pioneering studies on the concept of the protective layer without arginine catalysis. The authors also wish to thank Dr James Sullivan's group (University College Dublin) for the use of the IR spectrophotometer, Rui Zhang and Dr Munuswamy Venkastesan (Trinity College Dublin) for their help with the magnetic measurements and Dr Ruth Doherty (CBNI) for her kind support with the preparation of the manuscript. Niamh O'Raw and Sophie Fernan are also acknowledged for their supporting work.

\section{References}

1 K. A. Dawson and Y. Yan, Nat. Nanotechnol., 2021, 16, 229242.

2 E. Sheridan, S. Vercellino, L. Cursi, L. Adumeau, J. A. Behan and K. A. Dawson, Nanoscale Adv., 2021, 3, 2397-2410.

3 W. Wu, J. Changzhong and V. A. L. Roy, Nanoscale, 2015, 7, 38-58.

4 D. Alex, A. Mathew and R. K. Sukumaran, Bioresour. Technol., 2014, 167, 547-550.

5 K. Li, Y. Fan, Y. He, L. Zeng, X. Han and Y. Yan, Sci. Rep., 2017, 7, 16473.

6 E. Amstad, S. Zurcher, A. Mashaghi, J. Y. Wong, M. Textor and E. Reimhult, Small, 2009, 5, 1334-1342.

7 J.-s. Choi, S. Kim, D. Yoo, T.-H. Shin, H. Kim, M. D. Gomes, S. H. Kim, A. Pines and J. Cheon, Nat. Mater., 2017, 16, 537-542.

8 S. M. Dadfar, K. Roemhild, N. I. Drude, S. von Stillfried, R. Knüchel, F. Kiessling and T. Lammers, Adv. Drug Delivery Rev., 2019, 138, 302-325.

9 I. Rubia-Rodríguez, A. Santana-Otero, S. Spassov, E. Tombácz, C. Johansson, P. De La Presa, F. J. Teran, M. d. P. Morales, S. Veintemillas-Verdaguer, N. T. K. Thanh, M. O. Besenhard, C. Wilhelm, F. Gazeau, Q. Harmer, E. Mayes, B. B. Manshian, S. J. Soenen, Y. Gu, Á. Millán, E. K. Efthimiadou, J. Gaudet, P. Goodwill, J. Mansfield, U. Steinhoff, J. Wells, F. Wiekhorst and D. Ortega, Materials, 2021, 14, 706.

10 P. Guardia, R. Di Corato, L. Lartigue, C. Wilhelm, A. Espinosa, M. Garcia-Hernandez, F. Gazeau, L. Manna and T. Pellegrino, ACS Nano, 2012, 6, 3080-3091.

11 E. A. Périgo, G. Hemery, O. Sandre, D. Ortega, E. Garaio, F. Plazaola and F. J. Teran, Appl. Phys. Rev., 2015, 2, 041302.

12 M. K. Masud, J. Na, M. Younus, M. S. A. Hossain, Y. Bando, M. J. A. Shiddiky and Y. Yamauchi, Chem. Soc. Rev., 2019, 48, 5717-5751.

13 F. Bertoli, G. L. Davies, M. P. Monopoli, M. Moloney, Y. K. Gun'ko, A. Salvati and K. A. Dawson, Small, 2014, 10, 3307-3315.

14 H. M. Pezzi, D. J. Niles, J. L. Schehr, D. J. Beebe and J. M. Lang, ACS Omega, 2018, 3, 3908-3917. 
15 H.-W. Chen, Z.-S. Fang, Y.-T. Chen, Y.-I. Chen, B.-Y. Yao, J.-Y. Cheng, C.-Y. Chien, Y.-C. Chang and C.-M. J. Hu, ACS Appl. Mater. Interfaces, 2017, 9, 39953-39961.

16 M. I. J. Denison, S. Raman, N. Duraisamy, R. M. Thangavelu, S. U. M. Riyaz, D. Gunasekaran and K. Krishnan, RSC Adv., 2015, 5, 99820-99831.

17 B. Banik, B. W. Askins and S. Dhar, Nanoscale, 2016, 8, 19581-19591.

18 C. V. Mura, M. I. Becker, A. Orellana and D. Wolff, J. Immunol. Methods, 2002, 260, 263-271.

19 E. Ximendes, R. Marin, Y. Shen, D. Ruiz, D. Gómez-Cerezo, P. Rodríguez-Sevilla, J. Lifante, P. X. Viveros-Méndez, F. Gámez, D. García-Soriano, G. Salas, C. Zalbidea, A. Espinosa, A. Benayas, N. García-Carrillo, L. Cussó, M. Desco, F. J. Teran, B. H. Juárez and D. Jaque, Adv. Mater., 2021, 33, 2100077.

20 T. Fontecave, M. Bourbousson, C. Chaneac, C. Wilhelm, A. Espinosa, M.-A. Fortin, C. Sanchez and C. Boissiere, New J. Chem., 2016, 40, 4436-4446.

21 J. Reguera, D. J. d. Aberasturi, M. Henriksen-Lacey, J. Langer, A. Espinosa, B. Szczupak, C. Wilhelm and L. M. Liz-Marzán, Nanoscale, 2017, 9, 9467-9480.

22 F. Bertoli, D. Garry, M. P. Monopoli, A. Salvati and K. A. Dawson, ACS Nano, 2016, 10, 10471-10479.

23 M. B. Cutrona and J. C. Simpson, Small, 2019, 15, 1902033.

24 S. Lara, F. Alnasser, E. Polo, D. Garry, M. C. Lo Giudice, D. R. Hristov, L. Rocks, A. Salvati, Y. Yan and K. A. Dawson, ACS Nano, 2017, 11, 1884-1893.

25 A. Ditsch, S. Lindenmann, P. E. Laibinis, D. I. C. Wang and T. A. Hatton, Ind. Eng. Chem. Res., 2005, 44, 6824-6836.

26 S. Gyergyek, D. Makovec, A. Mertelj, M. Huskić and M. Drofenik, Colloids Surf., A, 2010, 366, 113-119.

27 S. A. Ansari, E. Ficiarà, F. A. Ruffinatti, I. Stura, M. Argenziano, O. Abollino, R. Cavalli, C. Guiot and F. D’Agata, Materials, 2019, 12, 465.

28 H. Ow, D. R. Larson, M. Srivastava, B. A. Baird, W. W. Webb and U. Wiesner, Nano Lett., 2005, 5, 113-117.

29 A. Van Blaaderen and A. Vrij, Langmuir, 1992, 8, 29212931.

30 V. Gubala, G. Giovannini, F. Kunc, M. P. Monopoli and C. J. Moore, Cancer Nanotechnol., 2020, 11, 1.

31 S. Foglia, M. Ledda, D. Fioretti, G. Iucci, M. Papi, G. Capellini, M. G. Lolli, S. Grimaldi, M. Rinaldi and A. Lisi, Sci. Rep., 2017, 7, 46513.

32 S. A. McCarthy, G.-L. Davies and Y. K. Gun'ko, Nat. Protoc., 2012, 7, 1677-1693.

33 J. Wan, X. Meng, E. Liu and K. Chen, Nanotechnology, 2010, 21, 235104.

34 O. Chen, L. Riedemann, F. Etoc, H. Herrmann, M. Coppey, M. Barch, C. T. Farrar, J. Zhao, O. T. Bruns, H. Wei, P. Guo, J. Cui, R. Jensen, Y. Chen, D. K. Harris, J. M. Cordero, Z. Wang, A. Jasanoff, D. Fukumura, R. Reimer, M. Dahan, R. K. Jain and M. G. Bawendi, Nat. Commun., 2014, 5, 5093.

35 L. Adumeau, C. Genevois, L. Roudier, C. Schatz, F. Couillaud and S. Mornet, Biochim. Biophys. Acta, Gen. Subj., 2017, 1861, 1587-1596.
36 C. J. Moore, G. Giovannini, F. Kunc, A. J. Hall and V. Gubala, J. Mater. Chem. B, 2017, 5, 5564-5572.

37 G. Battistelli, A. Cantelli, G. Guidetti, J. Manzi and M. Montalti, WIREs Nanomed. Nanobiotechnol., 2016, 8, 139-150.

38 S.-A. Yang, S. Choi, S. M. Jeon and J. Yu, Sci. Rep., 2018, 8, 185.

39 E. Mahon, D. R. Hristov and K. A. Dawson, Chem. Commun., 2012, 48, 7970-7972.

40 E. Yammine, L. Adumeau, M. Abboud, S. Mornet, M. Nakhl and E. Duguet, Nanomaterials, 2021, 11, 147.

41 A. Burns, P. Sengupta, T. Zedayko, B. Baird and U. Wiesner, Small, 2006, 2, 723-726.

42 T. Fontecave, C. Sanchez, T. Azaïs and C. Boissière, Chem. Mater., 2012, 24, 4326-4336.

43 E. Bindini, Z. Chehadi, M. Faustini, P.-A. Albouy, D. Grosso, A. Cattoni, C. Chanéac, O. Azzaroni, C. Sanchez and C. Boissière, ACS Appl. Mater. Interfaces, 2020, 12, 13598-13612.

44 G. Canton, R. Riccò, F. Marinello, S. Carmignato and F. Enrichi, J. Nanopart. Res., 2011, 13, 4349-4356.

45 S. A. Corr, Y. P. Rakovich and Y. K. Gun'ko, Nanoscale Res. Lett., 2008, 3, 87.

46 T. Yokoi, Y. Sakamoto, O. Terasaki, Y. Kubota, T. Okubo and T. Tatsumi, J. Am. Chem. Soc., 2006, 128, 13664-13665.

47 K. D. Hartlen, A. P. T. Athanasopoulos and V. Kitaev, Langmuir, 2008, 24, 1714-1720.

48 D. R. Hristov, E. Mahon and K. A. Dawson, Chem. Commun., 2015, 51, 17420-17423.

49 V. M. Masalov, N. S. Sukhinina, E. A. Kudrenko and G. A. Emelchenko, Nanotechnology, 2011, 22, 275718.

50 A. M. Milosevic, L. Rodriguez-Lorenzo, S. Balog, C. A. Monnier, A. Petri-Fink and B. Rothen-Rutishauser, Angew. Chem., Int. Ed., 2017, 56, 13382-13386.

51 T. Tenuta, M. P. Monopoli, J. Kim, A. Salvati, K. A. Dawson, P. Sandin and I. Lynch, PLoS One, 2011, 6, e25556.

52 T. dos Santos, J. Varela, I. Lynch, A. Salvati and K. A. Dawson, Small, 2011, 7, 3341-3349.

53 P. Sandin, L. W. Fitzpatrick, J. C. Simpson and K. A. Dawson, ACS Nano, 2012, 6, 1513-1521.

54 T. Andrian, R. Riera, S. Pujals and L. Albertazzi, Nanoscale Adv., 2021, 3, 10-23.

55 M. J. Rust, M. Bates and X. Zhuang, Nat. Methods, 2006, 3, 793-796.

56 R. Heintzmann and T. Huser, Chem. Rev., 2017, 117, 13890-13908.

57 A. G. Godin, B. Lounis and L. Cognet, Biophys. J., 2014, 107, 1777-1784.

58 N. Gustafsson, S. Culley, G. Ashdown, D. M. Owen, P. M. Pereira and R. Henriques, Nat. Commun., 2016, 7, 12471.

59 S. Culley, K. L. Tosheva, P. M. Pereira and R. Henriques, Int. J. Biochem. Cell Biol., 2018, 101, 74-79.

60 A. Salvati, C. Åberg, T. dos Santos, J. Varela, P. Pinto, I. Lynch and K. A. Dawson, Nanomedicine, 2011, 7, 818-826. 
61 N. Reinhardt, L. Adumeau, O. Lambert, S. Ravaine and S. Mornet, J. Phys. Chem. B, 2015, 119, 6401-6411.

62 S. Mornet, J. Portier and E. Duguet, J. Magn. Magn. Mater., 2005, 293, 127-134.

63 S. Mornet, Synthèse et modification chimique de la surface de nanoparticules de maghémite à des fins d'applications biomédicales, $\mathrm{PhD}$ thesis, Université Sciences et Technologies, Bordeaux I, 2002.

64 G. A. van Ewijk, G. J. Vroege and A. P. Philipse, J. Magn. Magn. Mater., 1999, 201, 31-33.

65 D. L. Green, J. S. Lin, Y.-F. Lam, M. Z. C. Hu, D. W. Schaefer and M. T. Harris, J. Colloid Interface Sci., 2003, 266, 346-358. 